\title{
Influence on Intention to Adopt Green IS: Boosting Endogenous Motivations with Persuasive Systems Design
}

\author{
Nataliya Shevchuk \\ University of Oulu \\ nataliya.shevchuk@oulu.fi
}

\author{
Harri Oinas-Kukkonen \\ University of Oulu \\ harri.oinas-kukkonen@oulu.fi
}

\begin{abstract}
Because pro-environmental behavior is often perceived as burdensome, encouraging sustainable habits can be a challenging task. Green IS provide additional means to instill proper sustainable manners in its users. However, even adoption of Green IS is not necessarily easy and is likely to require both motivation and persuasion. In this paper, we analyze the impact of Persuasive Systems Design on endogenous motivations linked to the attitude formation and subsequent intention to adopt Green IS. Based on the presented theoretical background, we construct a research model capturing relationships among persuasive design categories, different types of motivations, attitude and intention to adopt Green IS. Using structural equation modeling, we analyze the data collected with the survey. Findings of our study prove that the researched concepts are interrelated showing impact of computerhuman dialogue support, system credibility support, and social support on extrinsic motivation and suggesting importance of enhancing Green IS with Persuasive Systems Design.
\end{abstract}

\section{Introduction}

In the era of omnipresent social web and extensive use of mobile applications, creating, accessing, and sharing information has become easier than ever before since the users of interactive information systems can be reached in a matter of seconds. Therefore, influencing users with various information systems, such as the web, the Internet, mobile, and other ambient systems, creates opportunities for persuasive interaction. Combining attributes of interpersonal and mass communication, web and mobile systems are ideal for persuasion $[6,46]$. Such interactive information systems designed for changing users' attitudes or behavior are known as persuasive systems, and Persuasive Systems Design
(PSD) is one of the frameworks for their development [46].

Up-to-date, fostering improved health and healthier lifestyles has dominated the area of application of persuasive systems. However, PSD has shown to be handy in evaluating and creating other types of systems, for instance Green IS which support sustainable behavior change $[4,8,55]$; yet this area of research remains less investigated and requires more attention.

Overall, benefits of using Green IS are manifold, ranging from lower expenditures or increased control of energy consumption to larger indirect benefits for society, such as lower greenhouse gas emissions [63]. Nevertheless, Green IS alone cannot achieve the positive impact without the individuals' motivation to acquire sustainable or pro-environmental behavior, i.e. behavior that harms the environment as little as possible, or even benefits the environment [56]. Oftentimes, such behavior is seen as not enjoyable because it is linked to personal disadvantages like behavioral constraints or loss of comfort [41]. Because behavior change is not typically fun and sometimes not voluntary, resistance to Green IS sustainability issues increases when changes in existing routines are required [3].

Since no universal theory of persuasion exists, we must draw from a set of theories and models that describe influence, motivation, or behavior change in specific situations and for specific types of people [21]. We propose that PSD is an approach that is likely to affect people's motivations and consequentially induce desired behavioral patterns. Most of the IS research so far has conceptualized motivation as being primarily exogenous, meaning that behavior is a result of external stimuli [63]. However, the subjective psychological meanings of these stimuli and the type of motivation, i.e. autonomous versus controlled, have shown to be even more important than the mere amount of motivation [14, 52]. Thus, we aim to research the following question:

RQ: How do endogenous motivations, influenced by $P S D$, shape intention to adopt Green IS?

Next, we present theoretical background of Green IS, PSD model and studying endogenous motivations 
through Organismic Integration Theory . After that, we construct a research framework that creates hypothetical connections among PSD categories, endogenous motivation, attitude towards Green IS and their adoption to help understand relationships of these constructs. Analyzing the data collected with the survey, we employ structural equation modeling to draw implications relevant for the answering the research question.

\section{Theoretical Background}

\subsection{Green IS}

Because environmental sustainability is "the issue of the day" [49] and "one of the most important global challenges of the 21 st century" [14, 40], the information systems discipline has both a responsibility and an opportunity to contribute to solving this challenge [61]. Green IT discusses mitigating adverse effects of IT on environment through more energy-efficient systems with minimized environmental impact [7, 17, 30]. More broadly, Green IS describes the utilization of technologies and systems that serve as "a potential enabler of green, sustainable solutions" [53, p. 1] and as potential enabler of behavioral change by individuals, organizations, and society [30].

Recently, Green IS has developed into a wide research field within the IS discipline. Green IS has considered sustainable practices of both individuals and organizations [16]. Initially, Green IS focused on business and industries in an attempt to emphasize how Green IS can become an integral part of business processes, how Green IS can develop firms' capabilities to adopt and practice sustainability, and how firms can design new techniques. On the organizational level, virtualization and remote work enable organizations to meet compliance imperatives and social norms related to organizational accountability for more environmentally responsible behaviors.

Since individuals can contribute to solving the problems of their societies [16], an important role of individuals' participation in addressing sustainability issues appeared in research. Individual actions were found to be central to shaping macro-level actions and initiatives. For example, beliefs-actions-outcomes (BAO) framework suggests that the individuals' beliefs contribute to shaping organizational and societal sustainable actions [40]. Moreover, environmental sustainability in relation to IT problems requires that IT professionals create knowledge and innovative Green IT solutions [42]. Employees' perceptions and leadership capabilities were also deemed to be crucial in the adoption of IS to support green initiatives [5, 57]. Regarding top-down initiatives, i.e. actions taken on the organizational level, managers' decisions have a great impact on compliance with sustainability requirements for green products [54].

Furthermore, Green IS research initiated consideration of user-centric solutions for sustainable improvements and development that encourage individuals choose more sustainable behaviors in their day-to-day routines [31].

\subsection{Persuasive Systems Design}

Persuasive Systems Design model is a tool developed for designing and evaluating Behavior Change Support Systems [45]. Behavior Change Support System (BCSS) is an instance of a persuasive system, and can be defined as a "sociotechnical information system with psychological and behavioral outcomes designed to form, alter or reinforce attitudes, behaviors or an act of complying without using coercion or deception" [45]. PSD process consists of three steps: (1) understanding the fundamental issues (postulates) behind persuasive systems before implementing the system, (2) analyzing the context and recognizing the intent, event, and strategies for the use of a persuasive system, and (3) designing actual qualities of a new persuasive system or evaluating features of an existing system [46]. This study focuses on the third step of the PSD process.

Designing qualities of a system is based on a wide range of software features classified in four categories: primary task support, computer-human dialog support, perceived system credibility support, and social support. Design principles of the primary task category, such as reduction, tailoring, tunneling, personalization, selfmonitoring, simulation, and rehearsal, focus on providing support for achieving primary goals of the user. Design principles related to computer-human dialog, e.g. rewards, praise, suggestions, reminders, similarity, liking, and social role, facilitate accomplishing established goal(s). Credibility support design principles, namely, trustworthiness, expertise, surface credibility, real-world feel, authority, third-party endorsements, and verifiability, aim to increase persuasiveness of the system by making it more credible. Design principles in the social influence category introduce system features that motivate users by leveraging social behaviors, such as recognition, competition, cooperation, normative influence, social learning, social comparison, and social facilitation. Examples of implementation of the persuasive features relevant to Green IS are reflected in items of the PSDrelated constructs (Appendix A). It is assumed that persuasive system features enhance participation and engagement with the interventions [32]. However, not all possible software features have to be present in a 
BCSS, because additional persuasive features may lead to decreased overall persuasiveness in some cases [45].

\subsection{Endogenous Motivations}

Endogenous motivations reflect how people's internal perceptions of autonomy, freedom, conflict, and feelings of external pressure affect intentions and behavior. Organismic Integration Theory (OIT) has been used in a number of scientific areas to explain the perceived degree of self-determination on behavior [14]. It provides a means to explain what the user experiences or feels and how this affects objectives and actions. The theory suggests that a person's behavior is driven by endogenous motivation, i.e a person volitionally initiates all behaviors instead of merely being triggered extrinsically by rewards or intrinsically when the activity itself is the reward (exogenous motivation) [14]. Thus, the theory views stimuli as affordances and opportunities used by a person for satisfying own needs, and not as direct causes of behavior.

OIT explains whether users feel autonomy, external pressure, or both by examining users' psychological states in terms of perceived locus of causality (PLOC). PLOC, defined as the degree to which actions are initiated from and endorsed by the individual [51], describes the relative autonomy of behavior. Users' feelings affect behavior regardless of the presence of external forces, i.e. users can feel compulsion even in absence of environmental pressures (e.g. behaving from guilt or obligation, rather than from own choice). Therefore, according to the theory, the user's perceptions of volition and compulsion are functions of PLOC rather than of external stimuli. OIT recognizes various feelings ranging from volition to compulsion, respectively characterized as internal and external PLOCs. Additionally, introjected PLOC explains a cumulative influence of both autonomy and external pressure on the behavior of a user willing to act in a manner different from the one forced on the user by the system. Figure 1 graphically represents how different types of PLOC and motivations match up.

\begin{tabular}{|c|c|c|}
\hline \multicolumn{2}{|c|}{ Extrinsic Motivation } & Intrinsic Motivation \\
\hline External & Introjected & \begin{tabular}{|l|l|} 
Identified PLOC & Intrinsic PLOC \\
\end{tabular} \\
\hline PLOC & PLOC & Internal PLOC \\
\hline
\end{tabular}

Figure 1. Endogenous motivations [39]

2.3.1. Internal PLOC. Internal PLOC consists of identified and intrinsic PLOCs with are associated with feelings of volition where actors perceive themselves as cause of their behavior. Intrinsic PLOC justifies instinctive and spontaneous behavior triggered by selfperceived reasons such as inherent enjoyment or fun [51]. Conversely, identified PLOC is characterized by behavior associated with the feelings of autonomy i.e. actions based on personal values and meaningful goals and outcomes [14]. Being rather volitional, identified PLOC is related to extrinsic motivation because it results from internalization and integration of external regulations adopted by individuals as personally important or valuable. Thus, identified PLOC pictures the individual's perception of the external regulation as self-regulation without interference of any immediate external consequences [14].

2.3.2. External PLOC. External PLOC is the least autonomous form of extrinsic motivation since it reflects completing actions under the influence of others [14]. In other words, it is associated with perceived reasons for behavior performed to satisfy an external demand or compliance to authority [51]. Because external PLOC relates to external regulation of behavior, it represents internalization and integration of the social influences. However, external PLOC assumes no conflict between perceived external influences and personal values of the user.

2.3.3. Introjected PLOC. Introjected PLOC is relevant when there is an apparent conflict caused by misalignment of the perceived external behavioral (social) influences and personal norms and values. Nevertheless, this extrinsic motivation spawns perceived reasons for one's behavior that are related to affective feelings of guilt and shame, and esteem-based pressures to act $[13,51]$. Thus, introjected PLOC is often associated with strong self-imposed feelings of coercion that can result into rejection of the behavior. Although both external PLOC and introjected PLOC are activated by external pressure, they represent distinct psychological states with different behavioral outcomes [51]. While external PLOC is typically met with negligible resistance resulting in compliance, introjected PLOC involves rather strong feelings of violation of personal values, compulsion, compliance, and tension, which may be self-administered even in the absence of any external pressures. Overall, perceived pressure, ambivalence, anxiety, and frustration of this state make behavior adoption more difficult. Thus, without viewing external influence as own internal value, the individual will experience difficulties with integrating social norms into own value system.

\section{Research Model and Hypotheses}

Based on the discussed theoretical background, we propose the following research model and hypotheses (Figure 2). Although the model could be tested in various contexts, we will focus on Green IS with 
persuasive features aimed to foster sustainable behavior. We predict that all constructs related to the PSD model are likely to influence all types of PLOCs, because the persuasive support categories are likely to trigger different types of motivations and either cause alignment or misalignment with the personal norms. Additionally, prior research [39, 62, 63] showed that all types of PLOCs have influence on the individuals' attitudes, and therefore, we assume that these relationships will hold up in the current study as well. Moreover, as suggested by the theory of reasoned action (TRA) [20] and the theory of planned behavior (TPB) [1], we expect to see strong connections between attitude towards Green IS and intention to adopt them.

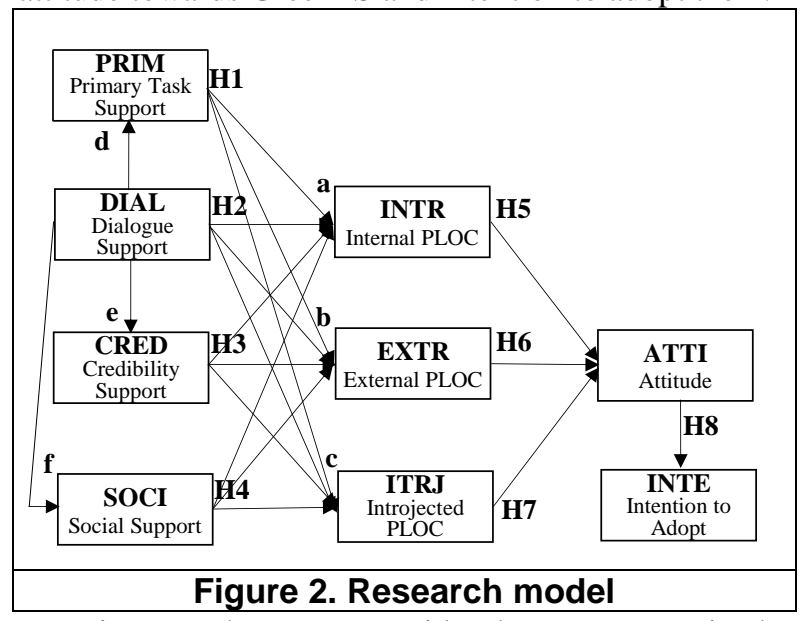

Primary task support provides the means to assist the user in carrying out the behavior. By enhancing selfefficacy of the user with the primary support features, cognitive burden and disorientation involved in using the system can be reduced [43]. Primary task support is related to cognitive fit [59], task-technology fit [26] and person-artefact-task fit [19]. It enables reflection on the individual's behavior, personal goal-setting and tracking progress towards the goals [38]. In the context of sustainable behavior, primary task support features can help overcome psychological barriers and reduce the perceived effort of engaging in environmental sustainable behavior that some people view as a burden [37]. Thus, we predict that if the system provides the means which assist in achieving sustainable behavior, people will be more motivated to engage in proenvironmental activities.

H1a: Primary task support influences internal PLOC H1b: Primary task support influences external PLOC H1c: Primary task support influences introjected PLOC

Dialogue support assists with keeping the user active and motivated to use the system, helping to perform target behavior. Ideally, dialogue support promotes users' positive affect, which will likely influence users' confidence in the source (credibility) [28, 34, 35, 36, 44]. Moreover, people tend to react to IT artefacts as if they are interacting in social situations [2]. Additionally, because people's social relationships are being increasingly maintained through technology-mediated communications, dialogue support is likely to influence social support [34, 44]. Therefore, we predict that as a result of the effective system-human interaction, the users' motivation to participate in sustainable behavior is likely to increase. Furthermore, communication with the system has been shown to impact features that support engaging in the primary task, system's credibility [28, 35, 36, 44], and social interaction with the other users of the system [34, 44].

H2a: Dialogue support influences internal PLOC $H 2 b$ : Dialogue support influences external PLOC H2c: Dialogue support influences introjected PLOC H2d: Dialogue support influences primary task support H2e: Dialogue support influences credibility support H2f: Dialogue support influences social support

Credibility support attempts to strengthen the effect of persuasion by making the system more credible [46]. Perceived credibility can be achieved by providing endorsements from respected and renowned sources (e.g. a recommendation by an authoritative organization, an award for excellence in usability, or a privacy seal to ensure confidentiality). A highly credible source is usually perceived as more persuasive than a low-credibility one [50]. Trust is a closely related concept often discussed in IS research [35, 36]. Accepting the advice, trusting the information, and believing the output are signs of computer credibility [18]. Trust, belief, and credibility can have a significant impact on the users' expectations in performing target behavior [15]. People make initial assessments of the system's surface credibility based on the initial encounter [60]. Thus, perception of credibility is highly subjective and can vary significantly. If people find that information on environmental impacts and sustainable initiatives provided by the authorities lacks credibility [28], they are likely not to pursue sustainable behavior. Therefore, we hypothesize that if the system is overall perceived as trustworthy and reliable, people will be more willing to using the system and participate in proenvironmental actions encouraged by the system.

H3a: Credibility support influences internal PLOC

$H 3$ b: Credibility support influences external PLOC

H3c: Credibility support influences introjected PLOC

Social support design principles motivate users by leveraging social influence that is fundamental for proenvironmental mindset and behavior [25]. When feeling a necessity to join a community, some people will be open to adjust own behavior [48]. Opinions of friends, family and peers are highly likely to change one's view on adoption of sustainable behavior [25]. Social activities and interaction with the like-minded people with similar interests or personal goals can promote the 
users' favorable perception of Green IS and increase willingness to engage in sustainable behavior [37]. Thus, we hypothesize that social support features have a positive impact on increasing motivation to pursue sustainable behavior.

H4a: Social support influences internal PLOC

H4b: Social support influences external PLOC

H4c: Social support influences introjected PLOC

By definition, attitude is the individual's positive or negative feeling (evaluative affect) about performing certain behavior [20]. It reflects the individual's feeling that system adoption and use is experienced as positive (desirable) or negative (undesirable). Feelings of autonomy, volition, and freedom are expected to be associated with a positive attitude and desirability of the behavior. When individuals are finding the behavior personally important, they feel good about themselves and form a positive attitude towards the behavior. Thus, when behavioral motivation is associated with the perceived autonomy, it is expected to positively influence attitude [23].

\section{H5: Internal PLOC influences attitude}

Social rewards and contingencies, such as praise, approval, and social esteem may motivate external regulation and "promote certain otherwise nonspontaneous behaviors" [12, p. 135]. Under the influence of external PLOC, an individual is likely to associate the feelings towards performing the specific behavior to such external influences. Compared to internal PLOC, positive feelings associated with autonomy and choice are not so strong yet supported by the findings of the previous studies [62, 63], thus, we hypothesize that external PLOC will have an influence on the individual's attitudes. Unlike internal PLOC which supports personally meaningful and self-growthoriented activities, external PLOC is associated with the less meaningful activities which results in the lesser feelings of ease [9]. The excitement and enthusiasm typical for internal PLOC is lacking in behavior which is not fully self-endorsed [14], so the individuals motivated by external PLOC are less interested in the behavior. Despite of not being as enthusiastic, they are still likely to perform the behavior under the influence of a personally meaningful external incentive or reward perceiving the behavior as not overly burdensome, autonomous, and relatively easy to perform [14].

H6: External PLOC influences attitude

When users find themselves conflicted in doing what they perceive as mandated by social norms, unpleasant evaluative feelings may arise. This phenomenon is particularly common in the domain of eco-friendly behaviors as social influence is often the main cause of performing these behaviors since otherwise, an individual feels guilty or ashamed if not adopting or using Green IS. For example, if a person adopts or uses
Green IS under the influence of introjected PLOC the behavior is not the individual's volitional choice, but the behavior of others imposed on the individual [63]. In such case, the IS is used to increase the individual's selfesteem and to appeal to others who are deemed important. Hence, while proactively using Green IS or exercising pro-environmental practices that are important for the long-term well-being of the society, it may be peripheral to people's immediate "motives" causing pressure, ambivalence, anxiety, or frustration. The greater the conflict between personally meaningful goals and those they feel coerced into adopting, the more intense are the negative evaluative feelings (i.e. attitude) toward the specific behaviors. This leads to hypothesizing that introjected PLOC is likely to impact attitude.

\section{H7: Introjected PLOC influences attitude}

Prior research provides significant support for the impact of attitudes on the intention to adopt information technologies [29, 58], self-service technologies [10], and Green IS [33]. According to the Theory of Reasoned Action (TRA) [20], the Theory of Planned Behavior (TPB) [1], and the Technology Acceptance Model (TAM) [11], attitude of an individual influences intention, an essential component of performing a behavior. Hence, we predict that in line with previous research, the positive impact of attitude on behavioral intention will be supported in this study.

H8: Attitude influences intention to adopt sustainable behavior

\section{Research Method}

\subsection{Instrument Development}

The latent variables were measured using reflective multiple-item scales adopted with or without modifications for the context of the study from the prevalidated measures where possible (Appendix A). The items were measure using a 5-point Likert scale ranging from 'Not important' to 'Very important' to determine the extent to which the respondents perceived the importance of the described persuasive features, and a 7-point Likert scale ranging from 'Strongly disagree' to 'Strongly agree' to define the extent to which the respondents agreed with the statements regarding motivation, attitude and intention to adopt a Green IS. The 7-point Likert scale reflecting respondents' agreement was adopted from the previous studies (see Appendix A) using the same items, and the 5-point one was used to measure perceptions of importance of persuasive features, i.e. unique items developed for this study. Using a 5-point scale measurement simplifies reflecting perception of importance and reduces 
respondents' overall fatigue. A pilot survey was conducted among the experts on PSD who provided feedback on the questionnaire and helped refine the PSD-related items that were initially created to match the Green IS context of the study.

\subsection{Data Collection}

The distribution of the survey was implemented via an online software tool Webropol 2.0 and it was sent to the potential respondents (students of University of Oulu) via email containing an invitation to participate and the link to the questionnaire. Participants were asked to imagine an ideal (in their opinion) mobile application that could help them acquire proenvironmental behavior (i.e. Green IS). Without providing a bias of an excising application, this approach encouraged participants to brainstorm which persuasive features they considered to be relevant for achieving behavior change. In addition to questions related to the PSD categories, PLOCs, attitude and intent to adopt, demographic questions were asked. The link to the survey together with the invitation to participate in the study was emailed to 10,996 students of the University of Oulu. In total, 78 complete answers were obtained (response rate $.709 \%$ ), which contained no missing responses since all of the questions were set as mandatory. Descriptive statistics of the sample are provided in Table 1.

\begin{tabular}{|l|l|l|l|}
\hline \multicolumn{3}{|c|}{ Table 1. Descriptive statistics of the sample } \\
\hline Demographics & Value & $\#$ & $(\%)$ \\
\hline Age & $18-24$ & 35 & $45 \%$ \\
& $25-34$ & 37 & $47 \%$ \\
& $35-44$ & 3 & $4 \%$ \\
& $45-54$ & 3 & $4 \%$ \\
\hline Gender & Female & 50 & $64 \%$ \\
& Male & 28 & $36 \%$ \\
\hline Education & High school & 25 & $32 \%$ \\
& Bachelor's Degree & 41 & $53 \%$ \\
& Master's Degree & 11 & $14 \%$ \\
& Doctorate Degree & 1 & $1 \%$ \\
\hline Employment & Employed full-time & 5 & $6 \%$ \\
& Employed part-time & 6 & $8 \%$ \\
& Student & 67 & $86 \%$ \\
\hline
\end{tabular}

\section{Data Analysis and Results}

To discover which features the respondents perceived as the most important in a mobile app for assisting with leading pro-environmental lifestyle, we calculated mean values $(\mu)$ of each design principle (Appendix A). The results showed that the three highestrated features in each category were the following: reduction, simulation, self-monitoring in primary task support, suggestion, praise, liking in dialogue support, trustworthiness, expertise, surface credibility in credibility support, and competition, normative influence, and social comparison in social support. Comparing means among categories, the respondents found system credibility to be the most important $(\mu=$ $3.901)$, followed by primary task support $(\mu=3.533)$, dialogue support $(\mu=3.207)$, and social support $(\mu=$ 2.491)

Next, we used SmartPLS, a software with graphical user interface for variance-based structural equation modeling using the partial least squares path modeling method. PLS-SEM is used to predict, rather than to test established theory [27], so it suits well for exploratory research [24]. PLS-SEM minimum sample size should be equal to the larger of either (1) ten times the largest number of formative indicators used to measure one construct or (2) ten times the largest number of structural paths directed at a particular latent construct in the structural model [27]. Our sample size meets and exceeds the minimum requirements. Since the indicator data is routinely standardized in SmartPLS, measuring of different constructs with different scales (5- and 7point ones) does not constitute a problem. Testing the PLS-SEM model is carried out in two steps: assessment of the reliability and validity of the measurement model and assessment of the structural model. The measurement model includes the relationships between the constructs. The convergent and discriminant validity of the measurement instrument is examined to verify that the constructs' measures are valid and reliable before attempting to draw conclusions regarding relationships among constructs (i.e., structural model).

As all variables were measured using the same instrument, common method variance (CMV) or common method bias (CMB) is a potential threat to the validity of the results. To minimize CMV ex ante, the anonymity and confidentiality of the study invited the respondents to answer as honestly as possible. For the ex post test and possible control for CMV, a correlation matrix of the constructs was inspected to determine if any of the correlations were above .90 , which would serve as evidence that common method bias may exist [47]. In our case, none of the constructs correlated so highly. Full collinearity VIFs further indicate (all VIFs $<4)$ that CMV should not cause a detrimental effect (Appendix A).

\subsection{Assessment of Measurement Model}

The properties of the scales are assessed in terms of item loadings, discriminant validity, and internal consistency. Item loadings and internal consistencies greater than .70 are considered acceptable [22] (Appendix B). Therefore, the constructs in the model display good internal consistency, as evidenced by their 
composite reliability scores, with the lowest of .811 (PRIM) and the highest of .934 (INTE). In addition, AVE values of all the constructs were above the suggested minimum of .50 [22], thus demonstrating adequate internal consistency (Appendix B).

\subsection{Structural Model and Hypotheses Testing}

To evaluate the structural model, parametric bootstrapping with 5000 subsamples (parallel processing, no sign changes) was applied. The confidence interval method was the two-tailed biascorrected and accelerated bootstrap (the default setting). The path coefficients and explained variances $\left(\mathrm{R}^{2}\right)$ were obtained to assess the model. The path coefficients indicate the strength of the relationship of independent and dependent variables while $R^{2}$ measures the predictive power of the model for the dependent variables [27].

A blindfolding procedure was used to observe the predictive validity of the model. The Stone-Geisser cross-validated redundancy value $\left(\mathrm{Q}^{2}\right)$ above 0 is considered to indicate predictive validity of endogenous constructs. All endogenous constructs demonstrate $\mathrm{Q}^{2}>$ 0 , and thus indicate the path model's adequate predictive validity in connection with endogenous latent variables. $\mathrm{Q}^{2}$ is similar to $\mathrm{R}^{2}$ but is generally considered as a more reliable measure.

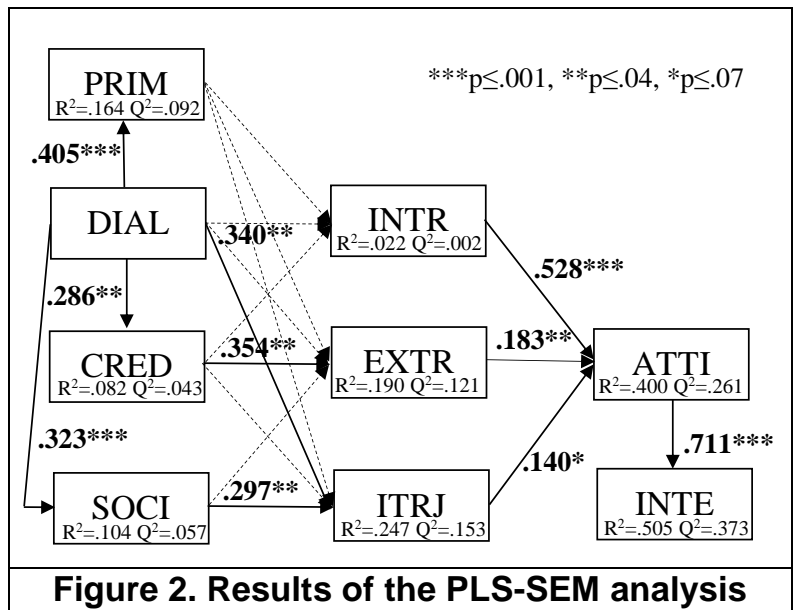

Based on the obtained results, in the structural model, DIAL explains $16 \%$ variance in PRIM, $8 \%$ in CRED and $10 \%$ in SOCI. Together PRIM, DIAL, CRED, and SOCI explain 19\% variance in EXTR, almost $25 \%$ in ITRJ, and $2 \%$ in INTR. INTR, EXTR, and ITRJ explain $40 \%$ variance in ATTI which alone explains $50.5 \%$ variance in INTE.

\section{Discussion}

We constructed and tested a theoretical research model which explains influence of the PSD model on different types of motivation and consequentially on attitude towards Green IS and intention to adopt Green IS. We conducted a survey which captured perceived importance of all persuasive features, the respondents' impressions on internal, external and introjected motivations, as well as attitude and intention to adopt Green IS. Comparing the means of the persuasive categories overall and the features individually, we determined that some are perceived as more important than the others, with the system credibility being the leading category. Results of the PLS-SEM analysis support a half of the hypotheses about relationships among the constructs. As expected, the persuasive system categories presented in the PSD model [46] interact in the predicted manner supported by findings of the previous studies [28, 34, 35, 36, 44]. Additionally, we discovered that dialog support impacts social support suggesting that the user's interaction with the system influence the user's interaction with the other users of the system. Moreover, the relationship between attitude and intention to adopt suggested by TRA [20] and TPB [1] and supported by multiple previous studies in IS research was significant in this study as well.

Regarding the endogenous motivation and the constructs in the Organismic Integration Theory, our findings were somewhat different from previous research. While internal and external PLOCs performed as expected in relation to attitude, introjected PLOC showed positive relationship to the user's attitude towards Green IS (instead of the negative one observed in the previous studies $[62,63])$. These findings suggest that extrinsic motivation (composed by both external and introjected PLOCs) just as intrinsic has a positive relationship on attitude towards Green IS. In line with the previous research, external PLOC showed a weaker influence. Furthermore, based on our findings regarding introjected PLOC, the respondents found factors shaping introjected PLOC to be not excessively forceful, and thus, affecting in a positive rather than a negative manner on forming attitude towards Green IS.

Analyzing the influence of PSD on different types of motivations, interesting findings emerged. None of the categories appeared to affect internal PLOC, suggesting that this type of motivation truly stems from the personal disposition of the individual and is difficult to be altered. Both dialogue and social support systems appeared to affect introjected PLOC, proposing that interaction with both the Green IS app and the other users of this IS impacts the person's extrinsic motivation even when it is introjected (i.e. does not align with the users personal beliefs). External PLOC was impacted only by 
credibility support, suggesting that the person's external regulation of behavior is strengthened by the perception of trust, believability, reliability, and credibility of Green IS, while nor the performance of the primary task or the interaction with the Green IS or its other users significantly impacted external PLOC.

\section{Conclusions}

Understanding what impacts people's decisions to use Green IS is crucial since it helps design more effective IS for sustainable behavior change. This study not only looked into possible enhancements that PSD categories can bring to Green IS but also into how they affect different types of motivations which ultimately shape the users' attitude towards Green IS and intention to adopt them. A theory-driven model and a measurement instrument were constructed and analyzed using PLS-SEM. The discussion of the results of the study provided possible explanations of the obtained findings and their relation to the existing research and practice. Considering the scope of available systems and apps, designers of Green IS need to identify which approaches are the most useful for achieving behavior change. Moreover, providers of Green IS are encouraged to recognize specific motivations of the users and choose specific persuasive techniques used in their systems accordingly to impact these motivations. This study offered insights into how adoption of Green IS is influenced by people's underlying needs and motivations.

The main contributions of the study include the constructed research model and development of the measurement instrument, both of which extend existing academic knowledge on adoption of Green IS and provide ideas for practitioners regarding how to design more persuasive and motivating Green IS. To our knowledge, this is the first study which simultaneously looks at both the PSD model and endogenous motivations explained with the Organismic Integration Theory. Although, the study is limited by the sample consisting only of the university students, the framework and the concepts can be applied to other setting and contexts.

Further research should consider a more detailed investigation of the individuals' motivations as well as their previous exposure to Green IS. Additionally, modifications to the research model and the measurement instrument can be considered. Using an existing system that the users interact with may provide further insights about the users' perceptions and behavior. Finally, surveying a more diverse sample could increase generalizability of the findings.

\section{References}

[1] I. Ajzen, "The theory of planned behavior," Organ. Behav. Hum. Decis. Process., vol. 50, no. 2, pp. 179-211, 1991.

[2] S. Al-Natour and I. Benbasat, "The Adoption and Use of IT Artifacts: A New Interaction-Centric Model for the Study of UserArtifact Relationships.," J. Assoc. Inf. Syst., vol. 10, no. 9, pp. 661-685, 2009.

[3] F. Bengtsson and P. J. Gerfalk, "Information technology as a change actant in sustainability innovation: Insights from Uppsala," J. Strateg. Inf. Syst., vol. 20, no. 1, pp. 96-112, 2011.

[4] B. Brauer, C. Ebermann, and L. M. Kolbe, "An Acceptance Model for User-Centric Persuasive Environmental Sustainable IS," Int. Conf. Inf. Syst. 2016, vol. 2, pp. 1-22, 2016.

[5] T. Butler, "Towards a Practice-Oriented Green IS Framework," ECIS, no. 2011, pp. 1-13, 2011.

[6]M. M. Cassell, C. Jackson, and B. Cheuvront, "Health communication on the Internet: an effective channel for health behavior change?," J. Health Commun., vol. 3, no. 1, pp. 71-79, 1998.

[7] A. J. Chen, R. T. Watson, and E. Karahanna, "Organizational Adoption of Green IS \& IT : An Institutional Perspective," ICIS, pp. 1-17, 2009.

[8] J. Corbett, "Designing and Using Carbon Management Systems to Promote Ecologically Responsible Behaviors," J. Assoc. Inf. Syst., vol. 14, no. 7, pp. 339-378, 2013.

[9] M. Csikszentmihalyi, "Beyond boredom and anxiety," B. Rev., pp. 703-707, 1975.

[10] J. M. Curran, M. L. Meuter, and C. F. Surprenant, "Intentions to Use Self-Service Technologies: A Confluence of Multiple Attitudes," J. Serv. Res., vol. 5, no. 3, pp. 209-224, 2003.

[11]F. D. Davis, R. P. Bagozzi, and P. R. Warshaw, "User Acceptance of Computer Technology: A Comparison of Two Theoretical Models," Manage. Sci., vol. 35, no. 8, pp. 982-1003, 1989.

[12] E. L. Deci and R. M. Ryan, "Conceptualizations of Intrinsic Motivation and Self-Determination," in Intrinsic Motivation and Self-Determination in Human Behavior, 1985, pp. 11-40.

[13] E. L. Deci, R. M. Ryan, and G. C. Williams, "Need satisfaction and the self-regulation of learning," Learn. Individ. Differ., vol. 8, no. 3, pp. 165-183, 1996.

[14] E. L. Deci and R. M. Ryan, Handbook of self-determination research. 2002.

[15] Y. K. Dwivedi, N. P. Rana, H. Chen, and M. D. Williams, "A Meta-analysis of the Unified Theory of Acceptance and Use of Technology (UTAUT)," in IFIP International Working Conf. on Governance and Sustainability in IS, 2011, pp. 155-170.

[16] S. C. El Idrissi and J. Corbett, "Green IS research: A modernity perspective," Commun. Assoc. Inf. Syst., vol. 38, no. 1, pp. 596-623, 2016.

[17] S. Elliot, "Transdisciplinary Prespectives on Environmental Sustainability: a Resource Base and Framework for It-Enabled Business Transformation," MIS Q., vol. 35, no. 1, pp. 197-236, 2011.

[18] A. Everard and D. F. Galletta, "How Presentation Flaws Affect Perceived Site Quality, Trust, and Intention to Purchase from an Online Store," J. Manag. Inf. Syst., vol. 22, no. 3, pp. 5695, 2006.

[19] C. M. Finneran and Z. Ping, "Flow in Computer-Mediated Environments: Promises and Challenges," Commun. AIS, vol. 2005, no. 15 , pp. 82-101, 2005.

[20] M. Fishbein and I. Ajzen, Belief, Attitude, Intention, and Behavior, An Introduction to Theory and Research, 1975.

[21] B. J. Fogg, Persuasive Technology: Using Computers to Change What We Think and Do. 2003.

[22] C. Fornell and D. Larcker, "Evaluating structural equation models with unobservable variables and measurement error," $J$. Mark. Res., vol. 18, no. 3, pp. 39-50, 1981. 
[23] M. Gagné and E. L. Deci, "Self-determination theory and work motivation," J. Organ. Behav., vol. 26, no. 4, pp. 331-362, 2005 .

[24] Gefen, Rigdon, and Straub, "Editor's Comments: An Update and Extension to SEM Guidelines for Administrative and Social Science Research," MIS Q., vol. 35, no. 2, p. iii, 2011.

[25] R. Gifford, "The Dragons of Inaction: Psychological Barriers That Limit Climate Change Mitigation and Adaptation," Am. Psychol., vol. 66, no. 4, pp. 290-302, 2011.

[26] D. L. Goodhue and R. L. Thompson, "Task-Technology Fit and Individual Performance," MIS Q., vol. 19, no. 2, p. 213, 1995 [27] J. F. Hair, C. M. Ringle, and M. Sarstedt, "PLS-SEM: Indeed a Silver Bullet," J. Mark. Theory Pract., vol. 19, no. 2, pp. 139152,2011

[28] K. Halttu and H. Oinas-Kukkonen, "Persuading to Reflect: Role of Reflection and Insight in Persuasive Systems Design for Physical Health," Human-Computer Interact., vol. 32, no. 5-6, pp. 381-412, 2017.

[29] M. H. Hsu and C. M. Chiu, "Internet self-efficacy and electronic service acceptance," Decis. Support Syst., vol. 38, no. 3, pp. 369-381, 2004.

[30] L. B. Iacobelli, R. A. Olson, and J. W. Merhout, "Green / Sustainable IT / IS : Concepts and Cases Green / Sustainable IT / IS : Concepts and Cases," AMCIS., p. 104, 2010.

[31] S. Y. Ijab, Mohamad T., Molla A. Kassahun, Asmare Emerie; and Teoh, "Seeking the 'Green' in 'Green IS': A Spirit, Practice and Impact Perspective," PACIS 2010, p. 46, 2010.

[32] S. M. Kelders, R. N. Kok, H. C. Ossebaard, and J. E. W. C. Van Gemert-Pijnen, "Persuasive system design does matter: A systematic review of adherence to web-based interventions," Journal of Med. Internet Res., vol. 14, no. 6. 2012.

[33] J. Kranz and A. Picot, "Why Are Consumers Going Green? the Role of Environmental Concerns in Private Green-Is Adoption," ECIS, no. 2011, p. Paper 104, 2011.

[34] T. Lehto and H. Oinas-Kukkonen, "Explaining and predicting perceived effectiveness and use continuance intention of a behaviour change support system for weight loss," Behav. Inf. Technol., vol. 34, no. 2, pp. 176-189, 2015.

[35] T. Lehto, H. Oinas-Kukkonen, and F. Drozd, "Factors affecting perceived persuasiveness of a behavior change support system," in ICIS, pp. 1-15, 2012.

[36] T. Lehto, H. Oinas-Kukkonen, T. Pätiälä, and O. Saarelma, "Consumer's perceptions of a virtual health check: An empirical investigation," in ECIS, 2012, p. Paper 154.

[37] S. Lindenberg and L. Steg, "Goal-framing theory and normguided environmental behavior," in Encouraging Sustainable Behavior: Psychology and the Environment, 2013, pp. 37-54.

[38] E. A. Locke and G. P. Latham, "Building a practically useful theory of goal setting and task motivation: A 35-year odyssey," Am. Psychol., vol. 57, no. 9, pp. 705-717, 2002.

[39] Y. Malhotra, D. F. Galletta, and L. J. Kirsch, "How Endogenous Motivations Influence User Intentions: Beyond the Dichotomy of Extrinsic and Intrinsic User Motivations," $J$. Manag. Inf. Syst., vol. 25, no. 1, pp. 267-300, 2008.

[40]N. P. Melville, "Information systems innovation for environmental sustainability," MIS $Q$., vol. 34, no. 1, pp. 1-21, 2010.

[41] C. J. H. Midden and B. S. M. Ritsema, "The meaning of normative processes for energy conservation," J. Econ. Psychol., vol. 4, no. 1-2, pp. 37-55, 1983.

[42] A. Molla, A. Abareshi, and V. Cooper, "Green IT beliefs and pro-environmental IT practices among IT professionals," Inf. Technol. People, vol. 27, no. 2, pp. 129-154, 2014.

[43] S. Nadkarni and R. Gupta, "A Task-Based Model Of Perceived Website Complexity," MIS Q., vol. 31, no. 3, pp. 501524, 2007.

[44] M. Oduor and H. Oinas-Kukkonen, "Commitment Devices as Behavior Change Support Systems: A Study of Users'
Perceived Competence and Continuance Intention," in Persuasive, 2017, pp. 201-213.

[45] H. Oinas-Kukkonen, "A foundation for the study of behavior change support systems," Pers. Ubiquitous Comput., vol. 17, no. 6, pp. 1223-1235, 2013.

[46] H. Oinas-Kukkonen and M. Harjumaa, "Persuasive systems design: Key issues, process model, and system features," Commun. Assoc. Inf. Syst., vol. 24, no. 1, pp. 485-500, 2009.

[47] Pavlou, Liang, and Xue, "Understanding and Mitigating Uncertainty in Online Exchange Relationships: A Principal-Agent Perspective," MIS Q., vol. 31, no. 1, p. 105, 2007.

[48] P. Petkov, F. Köbler, M. Foth, and H. Kramar, "Motivating domestic energy conservation through comparative, communitybased feedback in mobile and social media," in Intern.l Conf- on Commun. and Tech. - C\&T'11, 2011, p. 21.

[49] L. F. Pitt, M. Parent, I. Junglas, A. Chan, and S. Spyropoulou, "Integrating the smartphone into a sound environmental information systems strategy: Principles, practices and a research agenda," J. Strateg. Inf. Syst., vol. 20, no. 1, pp. 27-37, 2011. [50] C. Pornpitakpan, "The Persuasiveness of Source Credibility: A Critical Review of Five Decades' Evidence," J. Appl. Soc. Psychol., vol. 34, no. 2, pp. 243-281, 2004.

[51] R. M. Ryan and J. P. Connell, "Perceived locus of causality and internalization: Examining reasons for acting in two domains.," J. Pers. Soc. Psychol., vol. 57, no. 5, pp. 749-761, 1989.

[52] R. M. Ryan and E. L. Deci, "Intrinsic and Extrinsic Motivations: Classic Definitions and New Directions," Contemp. Educ. Psychol., vol. 25, no. 1, pp. 54-67, 2000.

[53] S. Seidel, J. Recker, and C. Pimmer, "Enablers and barriers to the organizational adoption of sustainable business practices," AMCIS 2010 Proc., no. August, pp. 12-15, 2010.

[54] S. Seidel, J. Recker, and J. vom Brocke, "Sensemaking and Sustainable Practicing: Functional Affordances of Information Systems in Green Transformations," MIS Q., vol. 37, no. 4, pp. 1275-1299, 2013.

[55] N. Shevchuk and H. Oinas-Kukkonen, "Exploring Green Information Systems and Technologies as Persuasive Systems : A Systematic Review of Applications in," ICIS., pp. 1-11, 2016.

[56] L. Steg and C. Vlek, "Encouraging pro-environmental behaviour: An integrative review and research agenda," $J$. Environ. Psychol., vol. 29, no. 3, pp. 309-317, 2009.

[57] B. Tan, S. L. Pan, and M. Zuo, "Harnessing collective IT resources for sustainability: Insights from the green leadership strategy of China mobile," J. Assoc. Inf. Sci. Technol., vol. 66, no. 4, pp. 818-838, 2015.

[58] S. Taylor and P. A. Todd, "Understanding information technology usage: A test of competing models," Inf. Syst. Res., vol. 6, no. 2, pp. 144-176, 1995.

[59] H. C. van Vugt, J. F. Hoorn, E. A. Konijn, and A. de Bie Dimitriadou, "Affective affordances: Improving interface character engagement through interaction," Int. J. Hum. Comput. Stud., vol. 64, no. 9, pp. 874-888, 2006.

[60] R. T. Watson, M.-C. Boudreau, and A. J. Chen, "Information systems and environmentally sustainable development: energy informatics and new directions for the is community," MIS Q., vol. 34, no. 1, pp. 23-38, 2010.

[61] I. Vessey and D. Galletta, "Cognitive fit: An empirical study of information acquisition," Inf. Syst. Res., vol. 2, no. 1, pp. 6384, 1991.

[62] P. Wunderlich, J. Kranz, D. Totzek, D. Veit, and A. Picot, "The Impact of Endogenous Motivations on Adoption of ITEnabled Services: The Case of Transformative Services in the Energy Sector,” J. Serv. Res., vol. 16, no. 3, pp. 356-371, 2013. [63] P. Wunderlich, J. Kranz, and D. Veit, "Beyond carrot-andstick: How values and endogenous motivations affect residential Green IS adoption,” ICIS, 2013, pp. 1-19. 


\section{Appendix A. Survey Instrument}

\begin{tabular}{|c|c|c|c|c|}
\hline Construct & Items & Mean & Loading & VIF \\
\hline $\begin{array}{l}\text { Primary } \\
\text { Task }\end{array}$ & $\begin{array}{l}\text { (Reduction) The app should decrease the complexity of my target behavior by breaking it into simpler } \\
\text { tasks. }\end{array}$ & 3.910 & 1- & - \\
\hline $\begin{array}{l}\text { Support } \\
{[46]}\end{array}$ & $\begin{array}{l}\text { (Tunneling) The app should guide me towards the target behavior by enabling only tasks that } \\
\text { contribute to it. }\end{array}$ & 3.244 & ]- & - \\
\hline (PRIM) & (Tailoring) The app should provide me information targeted to people like me. & 3.436 & - & \\
\hline & (Personalization) The app should provide individualized information targeted just for me. & 3.026 & & \\
\hline & (Self-monitoring) The app should help me track and monitor my behavior. & 3.782 & & \\
\hline & $\begin{array}{l}\text { (Simulation) The app should allow me to simulate the cause and effect of my behavior on the } \\
\text { environment. }\end{array}$ & 3.859 & .856 & 1.156 \\
\hline & (Rehearsal) The app should allow me to practice my target behavior. & 3.474 & .796 & 1.156 \\
\hline Dialogue & (Praise) The app should give positive feedback based on my behavior. & 3.808 & .873 & 2.009 \\
\hline Support & (Rewards) The app should reward me for achieving my goals. & 3.654 & .840 & 1.744 \\
\hline & (Reminders) The app should remind me of my goals and tasks to achieve. & 3.397 & .773 & 1.406 \\
\hline (DIAL) & (Suggestion) The app should provide me suggestions to help achieve my goals. & 3.949 & & \\
\hline & (Similarity) The app should imitate my personality. & 2.141 & & \\
\hline & (Liking) The app should appeal to me in terms of its look and feel. & 3.744 & & \\
\hline & $\begin{array}{l}\text { (Social role) The app should be based on a virtual character who would establish a personal } \\
\text { relationship with me. }\end{array}$ & 1.756 & & \\
\hline Credibility & (Trustworthiness) The app should be truthful, fair, and unbiased. & 4.577 & & - \\
\hline Support & (Expertise) The app should provide competent and up-to-date information. & 4.564 & & \\
\hline & (Surface credibility) The app should look professional. & 3.962 & .794 & \\
\hline$(\mathrm{CRED})$ & (Real-world feel) The app should provide information about the service provider. & 3.256 & & 1.178 \\
\hline & (Authority) The app should contain information provided by a trusted authority. & 4.013 & .868 & \\
\hline & (Third-party endorsements) The app should provide endorsements from external experts. & 3.064 & & 1.178 \\
\hline & $\begin{array}{l}\text { (Verifiability) The app should provide a means to verify the accuracy of its content via outside } \\
\text { sources. }\end{array}$ & 3.872 & & \\
\hline Social & (Social learning) The app should enable me to observe actions and outcomes of other people. & 2.462 & .885 & 1.871 \\
\hline Support & (Social comparison) The app should enable to compare my behavior with the behavior of others. & 2.744 & .794 & 1.514 \\
\hline [46] & (Normative influence) The app should suggest me what people are normally expected to do. & 2.795 & & \\
\hline (SOCI) & $\begin{array}{l}\text { (Social facilitation) The app should provide a means for figuring out who is performing the target } \\
\text { behavior along with me. }\end{array}$ & 2.282 & .842 & 1.785 \\
\hline & (Cooperation) The app should enable cooperation among the users. & 2.897 & & \\
\hline & (Competition) The app should enable competition among the users. & 2.410 & & \\
\hline & (Recognition) The app should give me public recognition for my behavior. & 1.846 & & \\
\hline External & I use/would use the app because it is recommended by my energy supplier. & 3.667 & .860 & 1.990 \\
\hline PLOC $[51$, & because it is recommended by governmental institutions. & 4.154 & .923 & 2.624 \\
\hline & because using the app offers me financial incentives. & 4.603 & & \\
\hline (EXTR) & because the European Union recommends using similar apps. & 4.026 & .877 & 2.334 \\
\hline Internal & I use/would use the app because I want to help protecting the environment. & 6.182 & & \\
\hline PLOC [51, & because I personally like using the app. & 5.013 & .872 & 2.490 \\
\hline 63] (INTR) & because I think it is personally important to myself. & 5.338 & .754 & 1.263 \\
\hline & because I want to learn how to use the app. & 3.532 & & \\
\hline & because I enjoy using the app. & 4.364 & .871 & 2.441 \\
\hline Introjected & I use/would use the app because I would feel bad about myself if I didn't use the app. & 2.974 & .834 & 1.604 \\
\hline PLOC $[51$, & because my peers think that I should use the app. & 3.038 & .903 & 3.104 \\
\hline 63] (ITRJ) & because of a current trend to do something to help protecting the environment. & 3.744 & & \\
\hline & because my friends think that I should use the app. & 3.000 & .872 & 2.623 \\
\hline & because I want my colleagues to like me. & 2.282 & & \\
\hline Attitude & I assume that it is a good idea to use the app. & 5.474 & .894 & 3.262 \\
\hline$[11,62]$ & I think that it is reasonable to use the app. & 5.385 & .852 & 2.700 \\
\hline (ATTI) & All in all, I think it is a bad idea to use the app. & 5.833 & .843 & 2.278 \\
\hline & I like the idea to use the app. & 5.295 & .876 & 2.619 \\
\hline Intention to & I would use the app in the future. & 5.051 & .859 & 2.418 \\
\hline Adopt [11, & I would be willing to try the app in the future. & 5.756 & .890 & 3.028 \\
\hline 34] (INTE) & I would consider using the app in the future. & 5.731 & .883 & 3.914 \\
\hline & I can imagine myself using the app in the future. & 5.372 & .897 & 3.658 \\
\hline
\end{tabular}

\section{Appendix B. Latent Variable Correlations}

\begin{tabular}{|c|c|c|c|c|c|c|c|c|c|c|c|}
\hline & CR & AVE & ATTI & IINTE & TCRED & DIAL & EXTR & ITRJ & INTR & |PRIM & SOCI \\
\hline ATTI & .923 & .751 & .867 & & & & & & & & \\
\hline INTE & .934 & .779 & .711 & .882 & & & & & & & \\
\hline CRED & .818 & .692 & .172 & .176 & .832 & & & & & & \\
\hline DIAL & 869 & .688 & .215 & .075 & .286 & .830 & & & & & \\
\hline EXTR & .917 & .787 & .329 & .267 & .395 & .266 & .887 & & & & \\
\hline ITRJ & .903 & .757 & .253 & .193 & .061 & .403 & .329 & .870 & & & \\
\hline INTR & .872 & .696 & .577 & .534 & .149 & .056 & .190 & .100 & .834 & & \\
\hline PRIM & 811 & .683 & .274 & .229 & .575 & .405 & .247 & .092 & .088 & .826 & \\
\hline SOCI & .879 & .707 & -.084 & -.014 & .145 & .323 & .191 & .395 & .033 & .093 & .841 \\
\hline
\end{tabular}

